\title{
Approximate differentiation: Jarník points
}

\author{
by
}

\author{
Jan Malý and Luděk Zajíček (Praha)
}

\begin{abstract}
We investigate Jarník's points for a real function $f$ defined in $\mathbb{R}$, i.e. points $x$ for which $\lim \operatorname{ap}_{y \rightarrow x}|(f(y)-f(x)) /(y-x)|=+\infty$. In 1970, Berman has proved that the set $J_{f}$ of all Jarník's points for a path $f$ of the one-dimensional Brownian motion is the whole $\mathbb{R}$ almost surely. We give a simple explicit construction of a continuous function $f$ with $J_{f}=\mathbb{R}$. The main result of our paper says that for a typical continuous function $f$ on $[0,1]$ the set $J_{f}$ is c-dense in $[0,1]$.
\end{abstract}

0. Introduction and notation. For an arbitrary real function $f$ of a real variable the set of points $x$ where $f_{\mathrm{ap}}^{\prime}(x)=+\infty$ is of measure zero, and so is the set of $x$ at which $\lim _{y \rightarrow x}|(f(y)-f(x)) /(y-x)|=+\infty$. Surprisingly, the "natural joint generalization" of these facts does not hold. In fact, Jarník [4] has constructed a continuous function $f$ such that $\lim \operatorname{ap}_{y \rightarrow x}|(f(y)-f(x)) /(y-x)|=+\infty$ for almost all $x$ and a function $g$ of Baire class 2 such that $\lim _{y \rightarrow x}|(g(y)-g(x)) /(y-x)|=+\infty$ for each $x$.

We shall say that $x$ is a Jarnik point for $f$ if

$$
\operatorname{limap}_{y \rightarrow x}\left|\frac{f(y)-f(x)}{y-x}\right|=+\infty
$$

and the set of all Jarník points for $f$ will be denoted by $J_{f}$. Almost forty years after Jarník's article, in the theory of stochastic processes [1] (cf. $[2]$ ) it was proved that almost every path of the one-dimensional Brownian motion serves as an example of a continuous function such that $J_{f}=\mathcal{R}$. In the first section of our paper we construct a continuous function with $J_{f}=\mathcal{R}$ as the sum of an explicitly defined trigonometric series. In fact, our example is slightly stronger, since we show that instead of the difference quotient $|(f(y)-f(x)) /(y-x)|$ it is possible to consider the quotient $\mid f(y)-$ $f(x) \mid / \varphi(|y-x|)$, where $\varphi$ is any prescribed increasing continuous function on $[0,+\infty)$ with $\varphi(0)=0$. 
The above fact that for a typical Brownian motion path $f$ we have $J_{f}=$ $\mathcal{R}$ naturally motivates the following question: What can be said about $J_{f}$ for a typical continuous function in $C([0,1])$ ? A result of the same article [4] of Jarník immediately gives that $\mu J_{f}=0$ for a typical function in $C([0,1])$. Theorem 2 of [10] implies an improvement of this fact: $J_{f}$ is $\sigma$-porous (and thus also a first category set) for a typical function in $C([0,1])$. On the other hand, the main result of the present paper contained in Section 3 says that $J_{f}$ is c-dense in $[0,1]$ for a typical function in $C([0,1])$. The proof is not easy, it is similar to Saks' [7] proof of the fact that a typical function in $C([0,1])$ is not a Besicovitch function. It has, similarly to Saks' proof, two basic ingredients:

(a) The Banach-Mazur game method which was in fact essentially used by Saks (cf. note on p. 103 of [9]).

(b) A basic lemma concerning general real functions.

Our basic lemma is proved in Section 2. We believe that it is also of independent interest since it gives an alternative proof of Theorem 9.7 of [8] which has a connection with the theory of the Denjoy integral.

In the following $\mu$ denotes the Lebesgue measure on the set $\mathcal{R}$ of all real numbers. We denote by $C$ the Banach space $C([0,1])$ equipped with the supremum norm. We say, as usual, that a typical function in $C$ has a property if the family of functions which do not have this property is a first category set. If $a \neq b$ are real numbers we denote by $\operatorname{co}(a, b)$ the convex hull of $\{a, b\}$, i.e. $\operatorname{co}(a, b)=[a, b]$ if $a<b$ and $\operatorname{co}(a, b)=[b, a]$ if $b<a$. Recall that

$$
J_{f}=\left\{x: \operatorname{limap}_{y \rightarrow x}\left|\frac{f(y)-f(x)}{y-x}\right|=\infty\right\} .
$$

1. A continuous function with $J_{f}=\mathcal{R}$. Recall that the first proof of the existence of such a function is probably contained in [1], where it is shown that a typical Brownian path serves as an example.

For our simple explicit construction we need the folowing easy lemma.

Lemma 1. Let $I$ be an interval of length $p>0, M \subset I$ and $0<\alpha<1$. Suppose that

$$
\left|\sin \frac{2 \pi x}{p}-\sin \frac{2 \pi y}{p}\right| \leq \alpha
$$

for every $x, y \in M$. Then

$$
\mu(M) \leq \frac{3 p}{\pi} \arccos (1-\alpha) .
$$


Proof. Let $J$ be an interval of the form $[k p / 4,(k+1) p / 4]$, where $k$ is an integer. Then clearly

$$
\mu(M \cap J) \leq \frac{p}{2 \pi} \mu\{x \in[0, \pi / 2]: \sin x \geq 1-\alpha\}=\frac{p}{2 \pi} \arccos (1-\alpha) .
$$

Since $I$ is contained in a union of 6 intervals of this type, we conclude that

$$
\mu(M) \leq 6 \frac{p}{2 \pi} \arccos (1-\alpha) .
$$

TheOREM 1. Let $\varphi:[0, \infty) \rightarrow[0, \infty)$ be an increasing continuous function with $\varphi(0)=0$. Then there exists a continuous function $f$ on $\mathcal{R}$ such that

$$
\operatorname{limap}_{y \rightarrow x} \frac{|f(y)-f(x)|}{\varphi(|y-x|)}=\infty \quad \text { for each } x \in \mathcal{R} .
$$

Proof. Put $\psi=\sqrt{\varphi}$. It is easy to see that if

(2) each $z \in \mathcal{R}$ is a density point for the set $\{x:|f(x)-f(z)|>\psi(|x-z|)\}$, then (1) holds. Now find a sequence $\left(a_{n}\right)_{n=1}^{\infty}$ such that $a_{n}>0$ and

$$
\sum_{j=n+1}^{\infty} a_{j} \leq \frac{a_{n}}{2 n} \quad \text { for each natural } n .
$$

Further, define inductively a sequence $\left(p_{n}\right)_{n=1}^{\infty}$ such that $p_{n}>0$,

$$
\begin{aligned}
2 \psi\left((n-1) p_{n-1}\right) & \leq \frac{a_{n}}{n} \quad \text { for } n=2,3, \ldots, \\
2 \pi p_{n} \sum_{i=1}^{n-1} \frac{a_{i}}{p_{i}} & \leq \frac{a_{n}}{n} \quad \text { for } n=2,3, \ldots,
\end{aligned}
$$

$$
n p_{n} \searrow 0 \text {. }
$$

We shall prove that the function

$$
f(x)=\sum_{n=1}^{\infty} a_{n} \sin \frac{2 \pi x}{p_{n}}
$$

satisfies (2). Of course, (3) implies that $f$ is continuous on $\mathcal{R}$. Now write $f_{n}(x)=a_{n} \sin \left(2 \pi x / p_{n}\right), s_{n}(x)=\sum_{j=1}^{n} f_{j}(x), r_{n}(x)=\sum_{j=n+1}^{\infty} f_{j}(x)$ and consider an arbitrary $z \in \mathcal{R}$. We want to show that $z$ is a dispersion point for the set $S:=\{x:|f(x)-f(z)| \leq \psi(|x-z|)\}$. To this end choose an arbitrary $0<h<p_{1}$. By (6) there is a unique natural $n=n(h) \geq 2$ such that $n p_{n}<h \leq(n-1) p_{n-1}$. First we shall prove that

(7) $\quad \mu(I \cap S) / \mu(I) \leq \arccos (1-3 / n)$ for each interval $I \subset(z-h, z+h)$ of length $p_{n}$. 
To prove (7), consider arbitrary points $x, y \in I \cap S$. Using the definition of $S$ and (4) we have

$$
\begin{aligned}
|f(x)-f(y)| & \leq|f(x)-f(z)|+|f(y)-f(z)| \\
& \leq \psi(|x-z|)+\psi(|y-z|) \\
& \leq 2 \psi\left((n-1) p_{n-1}\right) \leq a_{n} / n .
\end{aligned}
$$

Using (5), we obtain

$$
\begin{aligned}
\left|s_{n-1}(x)-s_{n-1}(y)\right| & \leq|x-y| \sup _{t \in \mathcal{R}}\left|\left(s_{n-1}\right)^{\prime}(t)\right| \\
& \leq 2 \pi|x-y| \sum_{i=1}^{n-1} \frac{a_{i}}{p_{i}} \leq 2 \pi p_{n} \sum_{i=1}^{n-1} \frac{a_{i}}{p_{i}} \leq \frac{a_{n}}{n} .
\end{aligned}
$$

Further, (3) gives

$$
\left|r_{n}(x)-r_{n}(y)\right| \leq 2 \sum_{j=n+1}^{\infty} a_{j} \leq \frac{a_{n}}{n} .
$$

Since $f_{n}=f-r_{n}-s_{n-1}$, we have

$$
\begin{aligned}
& \left|\sin \frac{2 \pi x}{p_{n}}-\sin \frac{2 \pi y}{p_{n}}\right|=\frac{1}{a_{n}}\left|f_{n}(x)-f_{n}(y)\right| \\
& \quad \leq \frac{1}{a_{n}}\left(|f(x)-f(y)|+\left|s_{n-1}(x)-s_{n-1}(y)\right|+\left|r_{n}(x)-r_{n}(y)\right|\right) \leq \frac{3}{n} .
\end{aligned}
$$

Thus by Lemma 1 ,

$$
\mu(I \cap S) \leq \frac{3 p_{n}}{\pi} \operatorname{arc} \cos (1-3 / n),
$$

which gives (7). Since $\lim _{h \rightarrow 0+} n(h)=+\infty$ and $n p_{n}<h$, it is clear that (7) implies

$$
\lim _{h \rightarrow 0+} \frac{\mu(S \cap(z-h, z+h))}{2 h}=0,
$$

which completes the proof.

Remark 1. If we put

$$
a_{n}=1 /(n !)^{2} \quad \text { and } \quad p_{n}=1 /((n+2) !)^{5},
$$

then the conditions (3)-(6) are satisfied for $\varphi(t)=t$. Consequently, the sum of the trigonometric series

$$
f(x)=\sum_{n=1}^{\infty} \frac{1}{(n !)^{2}} \sin \left(2 \pi((n+2) !)^{5} x\right)
$$

is a continuous function with $J_{f}=\mathcal{R}$. 
2. The basic lemma. In Section 3 we shall need Corollary 1 below which estimates the increment of a continuous function on an interval. But we shall start with a more general Lemma 2 which estimates a measure of an image of a set and deals with general measurable functions. The reason is that Lemma 2 is closely connected with a result of [8] concerning the condition (D) for functions, and consequently may be of independent interest.

Lemma 2. Let $f$ be a measurable function defined on a closed interval $I \subset \mathcal{R}$, let $M \subset I$ be a measurable set, $K>0$ and $p>0$. Suppose that for each $x \in M$ there exists a closed interval $I^{x}, x \in I^{x} \subset I$, such that

$$
\mu\left\{y \in I^{x}:\left|\frac{f(y)-f(x)}{y-x}\right|<K\right\} \geq p \mu I^{x} .
$$

Then

$$
\mu^{*} f(M) \leq \frac{4 K}{p} \mu I .
$$

Proof. For each $x \in M$ choose an interval $I^{x}$ as above and set

$$
J^{x}=\left(f(x)-K \mu I^{x}, f(x)+K \mu I^{x}\right) .
$$

We may assume that $\mu^{*} f(M)>0$. Choose $\beta \in\left(0, \mu^{*} f(M)\right)$. Find a compact set $E \subset \bigcup_{x \in M} J^{x}$ such that $\mu E>\beta$ and a finite set $F \subset M$ such that the subfamily $\left(J^{x}\right)_{x \in F}$ still covers $E$. By an easy covering argument (see e.g. Lemma 2 of [3]) we can find a set $T \subset F$ such that the system $\left(J^{x}\right)_{x \in T}$ is pairwise disjoint and

$$
\sum_{x \in T} \mu J^{x}=\mu\left(\bigcup_{x \in T} J^{x}\right) \geq \frac{1}{2} \beta .
$$

On the other hand, $\mu J^{x}=2 K \mu I^{x}$ and by $(9), \mu f^{-1}\left(J^{x}\right) \geq p \mu I^{x}$. Thus

$$
\mu I \geq \sum_{x \in T} \mu f^{-1}\left(J^{x}\right) \geq \frac{p \beta}{4 K} .
$$

Letting $\beta \rightarrow \mu^{*} f(M)$ we obtain the desired inequality.

Remark 2. Suppose that the assumption of Lemma 2 is strengthened in the following sense: for each $x \in M$ and $\delta>0$ there exists an interval $I^{x}$ (depending also on $\delta$ ), $x \in I^{x} \subset I \cap(x-\delta, x+\delta)$, for which (9) holds. Then we can assert that

$$
\mu^{*} f(M) \leq \frac{4 K}{p} \mu M
$$

In fact, it is sufficient for each $\varepsilon>0$ to cover $M$ by a pairwise disjoint countable family of closed intervals $\left(H_{\alpha}\right)_{\alpha \in A}$ such that $H_{\alpha} \subset I$ and $\mu\left(\bigcup_{\alpha \in A} H_{\alpha}\right)$ $<\mu M+\varepsilon$ and use Lemma 2 on each $H_{\alpha}$. This gives a quite different proof 
of Theorem 9.7 of Saks [8] (for measurable functions). Finally, note that under this strengthened assumption the inequality (10) can be improved to $\mu^{*} f(M) \leq(2 K / p) \mu M$ (cf. Lemma 9.2 of [8]). We can obtain an independent proof repeating the arguments of the proof of Lemma 2 and using the Vitali covering theorem.

In the sequel we shall need the following immediate corollary of Lemma 2. In fact, in our application $B=\emptyset$, but we prefer a more general formulation since we believe it is of some independent interest.

COROLlary 1. Let $f$ be a continuous function on a closed interval $[a, b]$ and let $c>0, p>0$ and a countable set $B \subset[a, b]$ be given. Suppose that for each $z \in[a, b] \backslash B$ there exists $x \in[a, b] \backslash\{z\}$ such that

$$
\mu\left\{y \in \operatorname{co}(x, z):|f(y)-f(z)|<\frac{1}{4} c p|y-z|\right\} \geq p|x-z| .
$$

Then $f(b)-f(a) \leq c(b-a)$.

3. Jarník's points for typical continuous functions. The following lemma is an easy consequence of Corollary 1.

LEMMA 3. Let $f$ be a continuous function on a closed interval $[a, b]$ and let $p>0, \tau>0$ and a countable set $B \subset[a, b]$ be given. Suppose that $|f(b)-f(a)| \geq \tau|b-a|$. Then there exists $z \in[a, b] \backslash B$ such that for each $x \in[a, b], x \neq z$, we have

$$
\mu\left\{y \in \operatorname{co}(x, z):|f(y)-f(z)|<\frac{1}{6} \tau p|x-z|\right\}<p|x-z| .
$$

The following lemma has a quite easy proof which is omitted.

LEMMA 4. Let $f$ be a measurable real function, $z \in \mathcal{R}$, and let $a_{n} \nearrow z$, $b_{n} \searrow z$ be such that for all $n \in \mathcal{N}$

$$
\mu\left\{y \in \operatorname{co}(x, z):\left|\frac{f(y)-f(z)}{y-z}\right|<n\right\} \leq \frac{1}{n}|x-z|,
$$

whenever $x \in\left[a_{n}, a_{n+1}\right]$ or $x \in\left[b_{n+1}, b_{n}\right]$. Then

$$
\operatorname{limap}_{y \rightarrow z}\left|\frac{f(y)-f(z)}{y-z}\right|=+\infty .
$$

LEMma 5. Let $f, g$ be continuous functions on an interval $[c-3 \xi, c+3 \xi]$, $\xi>0$, and let $m \geq 2$ be a real number. Suppose that

$$
\begin{array}{ll}
f^{\prime}(x)=3(m-1) & \text { if } \xi<|x-c|<3 \xi, \\
f^{\prime}(x)=300 m^{2} & \text { if }|x-c|<\xi
\end{array}
$$


and $\|g-f\| \leq \xi$. Then there is $z \in[c-\xi, c+\xi]$ such that for each $x \in$ $[c-3 \xi, c+3 \xi]$ we have

$$
\mu\{y \in \operatorname{co}(x, z):|g(y)-g(z)| \leq 4 m|y-z|\} \leq \frac{|x-z|}{4 m} .
$$

Pro of. We have

$$
f(c+3 \xi)-f(c-3 \xi) \geq 2 \xi\left(300 m^{2}+6\right),
$$

and thus

$$
\begin{aligned}
g(c+3 \xi)-g(c-3 \xi) & \geq 600 m^{2} \xi+12 \xi-2 \xi>600 m^{2} \xi \\
& >96 m^{2} \mu((c-3 \xi, c+3 \xi)) .
\end{aligned}
$$

By Lemma 3 there is $z \in[c-3 \xi, c+3 \xi]$ such that for each $x \in[c-3 \xi, c+3 \xi]$ we have

$$
\mu\left\{y \in \operatorname{co}(x, z):|g(y)-g(z)| \leq \frac{96 m^{2}}{6 \cdot 4 m}|y-z|\right\} \leq \frac{|x-z|}{4 m} .
$$

It remains to show that $|z-c| \leq \xi$. Assume the contrary, e.g. that $z \in$ $(c+\xi, c+3 \xi]$ (the case $z \in[c-3 \xi, c-\xi)$ is symmetrical). We can obviously find an interval $L \subset[c+\xi, c+3 \xi]$ of length $\frac{1}{2} \xi$, e.g. one of the intervals of the form

$$
[c+k \xi / 2, c+(k+1) \xi / 2], \quad k \in\{2,3,4,5\},
$$

such that $\operatorname{dist}(z, L)>\frac{1}{2} \xi$. If $y \in L$, then

$$
\begin{aligned}
|g(y)-g(z)| & \leq|f(y)-f(z)|+2 \xi \leq 3(m-1)|y-z|+4|y-z| \\
& \leq 4 m|y-z| .
\end{aligned}
$$

Let $x$ be the endpoint of $L$ for which $L \subset \operatorname{co}(x, z)$. Then

$$
\mu\{y \in \operatorname{co}(x, z):|g(y)-g(z)| \leq 4 m|y-z|\} \geq \mu L>\frac{|x-z|}{4 m} .
$$

This is a contradiction which shows that $|z-c| \leq \xi$.

One of the main tools of the present paper is the Banach-Mazur game (see [5], [6]). It is the following infinite game between two players.

Let $P$ be a metric space and $Q \subset P$ a subset. In the first step the first player chooses an open ball $B\left(g_{1}, \delta_{1}\right)$. In the second step the second player chooses an open ball $B\left(f_{1}, \varepsilon_{1}\right) \subset B\left(g_{1}, \delta_{1}\right)$, in the third step the first player chooses an open ball $B\left(g_{2}, \delta_{2}\right) \subset B\left(f_{1}, \varepsilon_{1}\right)$, and so on. If $\bigcap_{i=1}^{\infty} B\left(f_{i}, \varepsilon_{i}\right) \subset Q$, then the second player wins. In the opposite case the first player wins. We need the following theorem, essentially due to Banach.

THEOREM BM. The second player has a winning strategy in the BanachMazur game iff $Q$ is a residual subset of $P$. 
This theorem is proved in [6] in the case $P=(0,1)$; the proof in the general situation ([5]) is essentially the same.

Theorem 2. For a typical $f \in C$, the set $J_{f}$ of points $x$ for which

$$
\operatorname{limap}_{y \rightarrow x}\left|\frac{f(y)-f(x)}{y-x}\right|=+\infty
$$

is $\mathbf{c}$-dense in $(0,1)$.

Proof. Obviously it is sufficient to prove that for each fixed interval $(a, b) \subset(0,1)$ with rational endpoints the set $J_{f} \cap(a, b)$ has cardinality c for a typical $f \in C$. Put $Q=\left\{f: \operatorname{card}\left(J_{f} \cap(a, b)\right)=\mathbf{c}\right\}$. By Theorem BM it is sufficient to find a winning strategy for the second player in the BanachMazur game for $P=C$ and $Q$. We shall describe such a strategy. Put $S_{n}=\{0,1\}^{n}$ and $S=\{0,1\}^{\mathcal{N}}$, where $\mathcal{N}$ is the set of all natural numbers. By our strategy the second player will construct in his $n$th move not only the functions $f_{n} \in C$ and $\varepsilon_{n}>0$, but also $2^{n}$ points $\left(z_{s}\right)_{s \in S_{n}}$ such that the following conditions hold (where we have put $I_{s}=\left[z_{s}-3 \varepsilon_{n}, z_{s}+3 \varepsilon_{n}\right]$, $\left.L_{s}=\left[z_{s}-2 \varepsilon_{n}, z_{s}+2 \varepsilon_{n}\right], q_{n}=300(n+1)^{2}\right)$ :

(11) $\overline{B\left(f_{n}, \varepsilon_{n}\right)} \subset B\left(g_{n}, \delta_{n}\right)$

(12) $\left\{I_{s}: s \in S_{n}\right\}$ are pairwise disjoint subintervals of $(a, b)$,

(13) for each $s \in S_{n}, f_{n}^{\prime}(x)=3 n$ if $\varepsilon_{n}<\left|x-z_{s}\right|<3 \varepsilon_{n}$ and $f_{n}^{\prime}(x)=q_{n}$ if $\left|x-z_{s}\right|<\varepsilon_{n}$

if $n \geq 2$, then for any $t=\left(s_{1}, \ldots, s_{n}\right) \in S_{n}$ and $s=\left(s_{1}, \ldots, s_{n-1}\right)$,

(14) $I_{t} \subset L_{s}$

(15) for each $z \in L_{t}, x \in I_{s} \backslash I_{t}$ and $f \in B\left(f_{n}, \varepsilon_{n}\right)$ we have

$$
\mu\{y \in \operatorname{co}(x, z):|f(y)-f(z)| \leq n|y-z|\} \leq \frac{1}{n}|x-z| .
$$

If we show that the second player can play according to this strategy in all moves, we will be over. In fact, (14) and (11) imply that $\bigcap_{n=1}^{\infty} B\left(f_{n}, \varepsilon_{n}\right)$ consists of a single function $f$ and, for each $s=\left(s_{1}, s_{2}, \ldots\right) \in S$, the set $\bigcap_{n=1}^{\infty} I_{\left(s_{1}, \ldots, s_{n}\right)}$ consists of one point $z_{s} \in(a, b)$. Lemma 4 (putting $\left[a_{n}, b_{n}\right]=$ $\left.I_{\left(s_{1}, \ldots, s_{n}\right)}\right),(15)$ and (14) easily imply that $z_{s} \in J_{f}$. Since (12) implies that $z_{s_{1}} \neq z_{s_{2}}$ for $s_{1} \neq s_{2}$, we have $f \in Q$. Thus suppose that a natural number $m$ is fixed, the second player has played $m-1$ moves and the first player has played $m$ moves such that the conditions (11)-(15) hold for each $n<m$. We know further for $m>1$ that $B\left(g_{m}, \delta_{m}\right) \subset B\left(f_{m-1}, \varepsilon_{m-1}\right)$. Our task is to construct $f_{m}, \varepsilon_{m}$ and $\left\{z_{s}\right\}_{s \in S_{m}}$ such that (11)-(15) hold for $n=m$.

If $m=1$, then (14) and (15) are trivially satisfied for $n=m$ and the construction of $f_{1}, \varepsilon_{1}, z_{(0)}$ and $z_{(1)}$ such that (11)-(13) are satisfied for $n=1$ 
is quite easy. Assume $m>1$. By Lemma 5, for every $s \in S_{m-1}$ there is $c_{s} \in\left[z_{s}-\varepsilon_{m-1}, z_{s}+\varepsilon_{m-1}\right]$ such that for each $x \in I_{s}$ we have

$$
\mu\left\{y \in \operatorname{co}\left(x, c_{s}\right):\left|g_{m}(y)-g_{m}\left(c_{s}\right)\right| \leq 4 m\left|y-c_{s}\right|\right\} \leq \frac{\left|x-c_{s}\right|}{4 m} .
$$

Find $\varepsilon_{m}>0$ such that

(17) $\left(4 m q_{m}+7\right) q_{m} \varepsilon_{m}<\frac{1}{4} \delta_{m}$,

(18) for every $x, z \in[0,1]$, if $|x-z| \leq\left(4 m q_{m}+7\right) \varepsilon_{m}$, then $\mid g_{m}(x)-$ $g_{m}(z) \mid<\frac{1}{4} \delta_{m}$.

Define

$$
E_{s}=\left[c_{s}-\left(4 m q_{m}+7\right) \varepsilon_{m}, c_{s}+\left(4 m q_{m}+7\right) \varepsilon_{m}\right] .
$$

Observe that $\delta_{m} \leq \varepsilon_{m}$ and therefore by (17) we have $E_{s} \subset L_{s}$. We define $f_{m}(x)=g_{m}(x)$ if $x \in[0,1] \backslash \bigcup_{s \in S_{n-1}} E_{s}$. If $s \in S_{m-1}$, then we define $f_{m}$ on $E_{s}$ by the following conditions:

(19) $f$ is linear on $\left[c_{s}-\left(4 m q_{m}+7\right) \varepsilon_{m}, c_{s}-\left(4 m q_{m}+6\right) \varepsilon_{m}\right]$ and on $\left[c_{s}+\right.$ $\left.\left(4 m q_{m}+6\right) \varepsilon_{m}, c_{s}+\left(4 m q_{m}+7\right) \varepsilon_{m}\right]$,

$$
\begin{gathered}
f_{m}(x)=g_{m}(x) \text { if } x \in\left\{c_{s}, c_{s}-\left(4 m q_{m}+7\right) \varepsilon_{m}, c_{s}+\left(4 m q_{m}+7\right) \varepsilon_{m}\right\}, \\
f_{m}^{\prime}(x)=q_{m} \quad \text { if } 3 \varepsilon_{m}<\left|x-c_{s}\right|<5 \varepsilon_{m} \\
f_{m}^{\prime}(x)=3 m \quad \text { if either }\left|x-c_{s}\right|<3 \varepsilon_{m} \\
\quad \text { or } 5 \varepsilon_{m}<\left|x-c_{s}\right|<\left(4 m q_{m}+6\right) \varepsilon_{m} .
\end{gathered}
$$

If $t=\left(s_{1}, \ldots, s_{m}\right) \in S_{m}$ and $s=\left(s_{1}, \ldots, s_{m-1}\right)$, we set

$$
z_{t}= \begin{cases}c_{s}+4 \varepsilon_{m} & \text { if } s_{m}=1, \\ c_{s}-4 \varepsilon_{m} & \text { if } s_{m}=0 .\end{cases}
$$

We want to prove (11)-(15) for $n=m$. Since the graphs of $g_{m \mid E_{s}}$ and consequently also of $f_{m \mid E_{s}}$ lie by (17)-(21) obviously in the rectangle $E_{s} \times\left[g\left(c_{s}\right)-\frac{1}{4} \delta_{m}, g\left(c_{s}\right)+\frac{1}{4} \delta_{m}\right]$ the property (11) holds. Recall that $E_{s} \subset L_{s}$ for each $s \in S_{m-1}$ and thus (12) and (14) are proved. The property (13) follows from (21).

It remains to prove (15). Fix $t=\left(s_{1}, \ldots, s_{m}\right) \in S_{m}$ and set $s=$ $\left(s_{1}, \ldots, s_{m-1}\right)$. Write

$$
\begin{gathered}
M_{s}=\left\{y \in I_{s}:\left|g_{m}(y)-g_{m}\left(c_{s}\right)\right| \leq 4 m\left|y-c_{s}\right|\right\}, \\
T_{s}=\left\{x \in[0,1]:\left(4 m q_{m}+6\right) \varepsilon_{m}<\left|x-c_{s}\right|<\left(4 m q_{m}+7\right) \varepsilon_{m}\right\} .
\end{gathered}
$$

Suppose that $z \in L_{t}, x \in I_{s} \backslash I_{t}$ and $f$ is a continuous function on $[0,1]$ with $\left\|f-f_{m}\right\|<\varepsilon_{m}$. Define

$$
Z=\{y \in \operatorname{co}(x, z):|f(y)-f(z)| \leq m|y-z|\} .
$$


We want to obtain

$$
\mu Z \leq \frac{1}{m}|x-z|
$$

First consider $y$ such that $|y-z|>\varepsilon_{m} / m$ and $\left|y-c_{s}\right| \leq\left(4 m q_{m}+6\right) \varepsilon_{m}$. Notice that the slope of $f_{m}$ between $y$ and $z$ is estimated from below by $3 \mathrm{~m}$. Hence

$$
\begin{aligned}
|f(y)-f(z)| & \geq\left|f_{m}(y)-f_{m}(z)\right|-2 \varepsilon_{m} \\
& >3 m|y-z|-2 m|y-z|=m|y-z| .
\end{aligned}
$$

Consequently $y \notin Z$.

Further, consider $y \in I_{s} \backslash\left(M_{s} \cup E_{s}\right)$. Then we have

$$
|y-z| \geq\left|y-c_{s}\right|-\left|z-c_{s}\right| \geq\left(4 m q_{m}+7\right) \varepsilon_{m}-6 \varepsilon_{m}>4 m q_{m} \varepsilon_{m}
$$

and

$$
\left|f_{m}(z)-f_{m}\left(c_{s}\right)\right| \leq 6 \varepsilon_{m} q_{m} \leq 4 m q_{m} \varepsilon_{m}<|y-z| .
$$

Since $g_{m}(y)=f_{m}(y)$ and $g_{m}\left(c_{s}\right)=f_{m}\left(c_{s}\right)$ we obtain

$$
\begin{aligned}
|f(y)-f(z)| & \geq\left|f_{m}(y)-f_{m}(z)\right|-2 \varepsilon_{m} \\
& \geq\left|g_{m}(y)-g_{m}\left(c_{s}\right)\right|-\left|f_{m}\left(c_{s}\right)-f_{m}(z)\right|-2 \varepsilon_{m} \\
& \geq\left|g_{m}(y)-g_{m}\left(c_{s}\right)\right|-|y-z|-|y-z| .
\end{aligned}
$$

Using $y \notin M_{s}$, we further obtain

$$
\begin{aligned}
\left|g_{m}(y)-g_{m}\left(c_{s}\right)\right| & >4 m\left|y-c_{s}\right| \geq 4 m|y-z|-4 m\left|z-c_{s}\right| \\
& \geq 4 m|y-z|-24 m \varepsilon_{m}>4 m|y-z|-|y-z|
\end{aligned}
$$

and consequently

$$
|f(y)-f(z)|>4 m|y-z|-3|y-z|>m|y-z| .
$$

Thus $y \notin Z$. To prove (22), we distinguish two cases.

(i) If $\left|x-c_{s}\right|<\left(4 m q_{m}+6\right) \varepsilon_{m}$, then we have proved that either $Z \subset$ $\left[z-\varepsilon_{m} / m, z\right]$ or $Z \subset\left[z, z+\varepsilon_{m} / m\right]$. Consequently,

$$
\mu Z \leq \varepsilon_{m} / m \leq|x-z| / m .
$$

(ii) If $\left|x-c_{s}\right| \geq\left(4 m q_{m}+6\right) \varepsilon_{m}$, then $|x-z| \geq 4 m q_{m} \varepsilon_{m}$ and we have proved that

$$
\begin{aligned}
Z & \subset\left(\left[z-\varepsilon_{m} / m, z+\varepsilon_{m} / m\right] \cup T_{s} \cup M_{s}\right) \cap \operatorname{co}(x, z) \\
& \subset\left[z-\varepsilon_{m} / m, z+\varepsilon_{m} / m\right] \cup T_{s} \cup \operatorname{co}\left(c_{s}, z\right) \cup\left(M_{s} \cap \operatorname{co}\left(x, c_{s}\right)\right) .
\end{aligned}
$$

Using (16) we have

$$
\begin{aligned}
\mu Z & \leq \frac{2 \varepsilon_{m}}{m}+2 \varepsilon_{m}+6 \varepsilon_{m}+\frac{\left|x-c_{s}\right|}{4 m} \\
& \leq 10 \varepsilon_{m}+\frac{|x-z|}{4 m}+\frac{6 \varepsilon_{m}}{4 m} \leq \frac{|x-z|}{m} .
\end{aligned}
$$




\section{References}

[1] S. M. Berman, Gaussian processes with stationary increments: Local times and sample function properties, Ann. Math. Statist. 41 (1970), 1260-1272.

[2] D. Geman and J. Horowitz, Occupation densities, Ann. Probab. 8 (1980), 1-67.

[3] M. de Guzmán, A general form of the Vitali theorem, Colloq. Math. 34 (1975), 69-72.

[4] V. Jarník, Sur les nombres dérivées approximatifs, Fund. Math. 22 (1934), 4-16.

[5] J. C. Oxtoby, The Banach-Mazur game and Banach category theorem, in: Contribution to the Theory of Games III, Ann. of Math. Stud. 39, Princeton 1957, 159-163.

[6] —, Measure and Category, Springer, New York 1980.

[7] S. Saks, On the functions of Besicovitch in the space of continuous functions, Fund. Math. 19 (1932), 211-219.

[8] —, Theory of the Integral, Monograf. Mat. 7, Warszawa 1937 (reprinted by Hafner Publ., New York).

[9] L. Zajíček, The differentiability structure of typical functions in $C[0,1])$, Real Anal. Exchange 13 (1987-88), 119, 103-106, 93.

[10] - Porosity, derived numbers and knot points of typical continuous functions, Czechoslovak Math. J. 39 (114) (1989), 45-52.

FACULTY OF MATHEMATICS AND PHYSICS (KMA)

CHARLES UNIVERSITY

SOKOLOVSKÁ 83

18600 PRAHA 8, CZECHOSLOVAKIA

Received 22 April 1991 